PAPER

\title{
A Nonlinear Observer via Pseudo-Formal Linearization for Both State and Measurement Equations of Nonlinear Scalar-Measurement Systems
}

\author{
Kazuo Komatsu ${ }^{1}$ and Hitoshi Takata ${ }^{2}$ \\ ${ }^{1}$ National Institute of Technology, Kumamoto College, 2659-2 Suya, Koshi, Kumamoto 861-1102, Japan \\ ${ }^{2}$ Kagoshima University, 1-21-40 Korimoto, Kagoshima 890-0065, Japan \\ E-mail: ${ }^{1}$ kaz@kumamoto-nct.ac.jp
}

\begin{abstract}
In this paper, we develop a nonlinear observer by applying a pseudo-formal linearization method of the polynomial type to both state and measurement equations for nonlinear systems. The given nonlinear system is linearized into an augmented linear system by pseudo-formal linearization based on the Taylor expansion. Furthermore, an augmented measurement vector that consists of polynomials of measurement data is introduced and is transformed into an augmented linear one by the pseudo-formal linearization. A linear system theory is applied to these linearized systems to design a new nonlinear observer. Numerical experiments indicate that the performance of the presented method is more improved than by the previous method.
\end{abstract}

Keywords: nonlinear system, pseudo-formal linearization, nonlinear observer, Taylor expansion, linearization function

\section{Introduction}

In the past few decades, nonlinear systems have received increasing attention since many methods here and throughout [1]-[5] have been proposed. One of the design techniques for nonlinear estimation problems is to adopt linearization approaches, and we have been studying formal linearization methods. In our previous work, we presented a pseudo-formal linearization [6] by means of a piecewise Taylor expansion and an automatic choosing function [7] for scalar systems, which can improve the accuracy of a formal linearization method, and applied it to a nonlinear filter.

In this paper we develop a pseudo-formal linearization for multidimensional nonlinear systems and synthesize a nonlinear observer as an application. Introducing a linearization function that consists of polynomials, a nonlinear system is linearized by the pseudo-formal linearization with respect to the formal linearization function based on a Taylor expansion considering up to higher-order terms. In order to improve the accuracy of the nonlinear observer, an augmented measurement vector [8] is introduced, and then the given nonlinear measurement equation is also linearized with respect to the formal linearization function by pseudo-formal linearization. Linear system theories can be applied to these linearized systems to design a nonlinear observer.

Numerical experiments show that the presented method is superior to the previous method [9].

\section{Statement of Problem}

Nonlinear dynamic and measurement equations are described by

$$
\begin{gathered}
\Sigma_{1}: \dot{\boldsymbol{x}}(t)=\boldsymbol{f}(\boldsymbol{x}(t)), \quad \boldsymbol{x}(0)=\boldsymbol{x}_{0} \in \mathbf{D} \\
y(t)=h(\boldsymbol{x}(t))
\end{gathered}
$$

where $t$ denotes time, $\cdot=d / d t, \boldsymbol{x}$ is an $n$-dimensional state vector, $\boldsymbol{f} \in R^{n}$ is a sufficiently smooth nonlinear vector-valued function and $\mathbf{D} \subset R^{n}$ is a domain of $\boldsymbol{x}$. $y$ is a scalar measurement and $h \in R$ is a nonlinear function and continuously differentiable. The problem is to determine the state of the nonlinear dynamic system from the given measurement data $y$.

\section{Pseudo-Formal Linearization}

In order to linearize the given nonlinear state differential equation in Eq. (1), first we introduce the vector-valued separable function

$$
C: \mathbf{D} \rightarrow R^{L}
$$


which is continuously differentiable, where $C=$ $\left[C_{1}, \cdots, C_{j}, \cdots, C_{L}\right]^{T}$ and $L$ is the number of selected elements of the state vector. Let $D$ be a domain of $C^{-1}$. For example, if $x_{1}$ is the element of $\boldsymbol{x}$ that gives the highest nonlinearity of Eq. (1) (see Eq. (26)), then

$$
C(\boldsymbol{x})=x_{1} \in D \subset R \quad(L=1)
$$

The domain $D$ is divided into $(M+1)$ subdomains:

$$
D=\bigcup_{k=0}^{M} D_{k}
$$

where

$$
D_{M}=D-\bigcup_{k=0}^{M-1} D_{k}
$$

and $C^{-1}\left(D_{0}\right) \ni 0 . D_{k}(0 \leq k \leq M-1)$ endowed with a lexicographic order is the Cartesian product

$$
D_{k}=\prod_{j=1}^{L}\left[a_{k j}, b_{k j}\right), \quad\left(a_{k j}<b_{k j}\right)
$$

We here introduce an automatic choosing function of the sigmoid type [7],

$$
\begin{gathered}
I_{k}(\zeta)=\prod_{j=1}^{L}\left\{1-\frac{1}{1+\exp \left(2 \mu\left(\zeta_{j}-a_{k j}\right)\right)}\right. \\
\left.-\frac{1}{1+\exp \left(-2 \mu\left(\zeta_{j}-b_{k j}\right)\right)}\right\},(0 \leq k \leq M-1) \\
I_{M}(\zeta)=1-\sum_{k=0}^{M-1} I_{k}(\zeta)
\end{gathered}
$$

so that

$$
\sum_{k=0}^{M} I_{k}(\zeta)=1
$$

where $\zeta$ is the vector consisting of the selected elements of the state vector, or

$$
\zeta=\left[\zeta_{1}, \cdots, \zeta_{L}\right]^{T}=C(\boldsymbol{x})
$$

and $\mu$ is a positive real value. $I_{k}(\zeta)$ is analytic and almost unity on $D_{k}$, otherwise it is almost zero (see Fig. 1).

Using this automatic choosing function, we present a pseudo-formal linearization for nonlinear systems to improve the accuracy of a conventional formal linearization method [9] by using a Taylor expansion considering up to higher-order terms as follows.

We define an $N$ th-order formal linearization function that consists of polynomials defined by

$$
\begin{array}{r}
\phi(\boldsymbol{x}) \equiv\left[x_{1}, x_{2}, \cdots, x_{n}, \frac{x_{1}^{2}}{2 !}, \frac{x_{1} x_{2}}{1 ! 1 !}, \cdots, \frac{x_{n}^{2}}{2 !}, \cdots\right. \\
\left.\cdots, \frac{x_{1}^{r_{1}} x_{2}^{r_{2}} \cdots x_{n}^{r_{n}}}{r_{1} ! r_{2} ! \cdots r_{n} !}, \cdots, \frac{x_{n}^{N}}{N !}\right]^{T}
\end{array}
$$

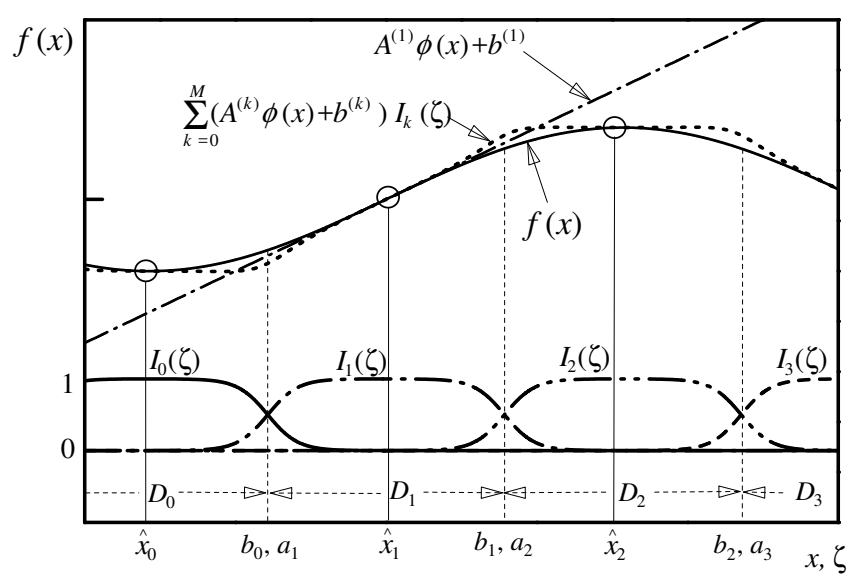

Fig.1 Pseudo-formal linearization using automatic choosing function

$$
=\left[\phi_{1}, \phi_{2}, \phi_{3}, \cdots, \phi_{\alpha_{\max }}\right]^{T}
$$

where $\alpha_{\max }$ is the number of combinations of $\left\{r_{1}, \cdots, r_{n}\right\}$ under the condition $r_{1}+\cdots+r_{n} \leq N$. Deriving the derivative of each element of $\phi$ along with the solution of the given nonlinear system (Eq. (1)), we obtain

$$
\begin{gathered}
\dot{\phi}_{\left(r_{1} \cdots r_{n}\right)}(\boldsymbol{x})=\frac{\partial}{\partial \boldsymbol{x}^{T}} \phi_{\left(r_{1} \cdots r_{n}\right)}(\boldsymbol{x}) \cdot \dot{\boldsymbol{x}} \\
=\frac{\partial}{\partial \boldsymbol{x}^{T}} \phi_{\left(r_{1} \cdots r_{n}\right)}(\boldsymbol{x}) \boldsymbol{f}(\boldsymbol{x}) \equiv f_{\left(r_{1} \cdots r_{n}\right)}^{*}(\boldsymbol{x})
\end{gathered}
$$

To each element of the linearization function, applying the Taylor expansion up to the $N$ th-order about a nominal operating point $\boldsymbol{x}=\hat{\boldsymbol{x}}_{k}$, where

$$
\hat{\boldsymbol{x}}_{k}=\left[\hat{x}_{1 k}, \cdots, \hat{x}_{n k}\right]^{T} \in D_{k}
$$

we have

$$
\begin{aligned}
\dot{\phi}_{\left(r_{1} \cdots r_{n}\right)}(\boldsymbol{x})=f_{\left(r_{1} \cdots r_{n}\right)}^{*}(\boldsymbol{x}) & \\
\approx \sum_{j_{1}=0}^{\left(j_{1}+\cdots+j_{n}\right)=N} & \left.\cdots \sum_{j_{n}=0}^{n} \frac{\partial^{\left(j_{1}+\cdots+j_{n}\right)}}{\partial x_{1}^{j_{1}} \cdots \partial x_{n}^{j_{n}}} f_{\left(r_{1} \cdots r_{n}\right)}^{*}(\boldsymbol{x})\right|_{\boldsymbol{x}=\hat{\boldsymbol{x}}_{k}} \\
& \times \prod_{i=1}^{n} \frac{\left(x_{i}-\hat{x}_{i k}\right)^{j_{i}}}{j_{i} !} \equiv \hat{f}_{\left(r_{1} \cdots r_{n}\right)}^{(k)^{*}}(\boldsymbol{x}) \\
= & \sum_{j_{1}=0}^{\left(j_{1}+\cdots+j_{n}\right)=N} \cdots \sum_{j_{n}=0}^{(k)\left(j_{1} \cdots j_{n}\right)} A_{\left(r_{1} \cdots r_{n}\right)}^{\left(j_{1} \cdots j_{n}\right)}(\boldsymbol{x})
\end{aligned}
$$

where

$$
A_{\left(r_{1} \cdots r_{n}\right)}^{(k)\left(j_{1} \cdots j_{n}\right)}=\left.\frac{\partial^{\left(j_{1}+\cdots+j_{n}\right)}}{\partial x_{1}^{j_{1}} \cdots \partial x_{n}^{j_{n}}} \hat{f}_{\left(r_{1} \cdots r_{n}\right)}^{(k)^{*}}(\boldsymbol{x})\right|_{\boldsymbol{x}=0}
$$

Namely,

$\dot{\phi}(\boldsymbol{x})_{\left(r_{1} \cdots r_{n}\right)} \approx\left[A_{\left(r_{1} \cdots r_{n}\right)}^{(k)(10 \cdots 0)}, A_{\left(r_{1} \cdots r_{n}\right)}^{(k)(01 \cdots 0)}, \cdots, A_{\left(r_{1} \cdots r_{n}\right)}^{(k)\left(j_{1} \cdots j_{n}\right)}\right.$, 


$$
\left.\cdots, A_{\left(r_{1} \cdots r_{n}\right)}^{(k)(0 \cdots N)}\right] \boldsymbol{\phi}(\boldsymbol{x})+A_{\left(r_{1} \cdots r_{n}\right)}^{(k)(0 \cdots 0)}
$$

Thus, it follows that on the subdomain $D_{k}$

$$
\dot{\boldsymbol{\phi}}(\boldsymbol{x}) \approx \boldsymbol{A}^{(k)} \boldsymbol{\phi}(\boldsymbol{x})+\boldsymbol{b}^{(k)}
$$

where

$$
\boldsymbol{A}^{(k)}=\left[A_{\left(r_{1} \cdots r_{n}\right)}^{(k)\left(j_{1} \cdots j_{n}\right)}\right], \boldsymbol{b}^{(k)}=\left[A_{\left(r_{1} \cdots r_{n}\right)}^{(k)(0 \cdots 0)}\right]
$$

We unite $(M+1)$ linearized systems (Eq. (12)) on subdomains into a single linear system on the whole domain by using Eq. (5) as

$$
\begin{gathered}
\dot{\phi}(\boldsymbol{x})=\sum_{k=0}^{M} \dot{\boldsymbol{\phi}}(\boldsymbol{x}) I_{k}(\zeta) \approx \sum_{k=0}^{M}\left(\boldsymbol{A}^{(k)} \boldsymbol{\phi}(\boldsymbol{x})+\boldsymbol{b}^{(k)}\right) I_{k}(\zeta) \\
=\tilde{A}(\zeta) \boldsymbol{\phi}(\boldsymbol{x})+\tilde{b}(\zeta)
\end{gathered}
$$

where

$$
\tilde{A}(\zeta)=\sum_{k=0}^{M} \boldsymbol{A}^{(k)} I_{k}(\zeta), \tilde{b}(\zeta)=\sum_{k=0}^{M} \boldsymbol{b}^{(k)} I_{k}(\zeta)
$$

Finally a pseudo-formal linearization system is defined as

$$
\Sigma_{2}: \dot{\boldsymbol{z}}(t)=\tilde{A}(\zeta) \boldsymbol{z}(t)+\tilde{b}(\zeta), \boldsymbol{z}(0)=\boldsymbol{\phi}(\boldsymbol{x}(0))
$$

From Eq. (7), its inversion is carried out by using

$$
\hat{\boldsymbol{x}}(t)=[I, 0, \cdots, 0] \boldsymbol{z}(t)
$$

as the approximated value of $\boldsymbol{x}(t)$, where $I$ is the $n \times n$ unit matrix.

\section{Nonlinear Observer}

Next we linearize the measurement equation (Eq. (2)) by the pseudo-formal linearization. To improve the accuracy of estimation, a new $m$ th-order measurement vector $\boldsymbol{Y}$ is introduced as

$$
\begin{gathered}
\boldsymbol{Y} \equiv\left[y, \frac{y^{2}}{2 !}, \cdots, \frac{y^{r}}{r !}, \cdots, \frac{y^{m}}{m !}\right]^{T} \\
=\left[h(\boldsymbol{x}), \frac{h^{2}(\boldsymbol{x})}{2 !}, \cdots, \frac{h^{r}(\boldsymbol{x})}{r !}, \cdots, \frac{h^{m}(\boldsymbol{x})}{m !}\right]^{T}
\end{gathered}
$$

We apply a Taylor expansion up to the $N$ th-order to each $\left\{\frac{h^{r}(\boldsymbol{x})}{r !}\right\}$ on the same subdomain $D_{k}$ as in a state space about a nominal operating point $\boldsymbol{x}=\hat{\boldsymbol{x}}_{k}$, then we have

$$
\begin{gathered}
Y_{r}=\frac{h^{r}(\boldsymbol{x})}{r !} \\
\left.\approx \sum_{j_{1}=0}^{\left(j_{1}+\cdots+j_{n}\right)=N} \cdots \sum_{j_{n}=0}^{n} \frac{\partial^{\left(j_{1}+\cdots+j_{n}\right)}}{\partial x_{1}^{j_{1}} \cdots \partial x_{n}^{j_{n}}} \frac{h^{r}(\boldsymbol{x})}{r !}\right|_{\boldsymbol{x}=\hat{\boldsymbol{x}}_{k}} \\
\times \prod_{i=1}^{n} \frac{\left(x_{i}-\hat{x}_{i k}\right)^{j_{i}}}{j_{i} !} \equiv \hat{h}_{r}^{(k)}(\boldsymbol{x})
\end{gathered}
$$

$$
=\sum_{j_{1}=0}^{\left(j_{1}+\cdots+j_{n}\right)=N} \cdots \sum_{j_{n}=0}^{N} H_{r}^{(k)\left(j_{1} \cdots j_{n}\right)} \phi_{\left(j_{1} \cdots j_{n}\right)}(\boldsymbol{x})
$$

where

$$
H_{r}^{(k)\left(j_{1} \cdots j_{n}\right)}=\left.\frac{\partial^{\left(j_{1}+\cdots+j_{n}\right)}}{\partial x_{1}^{j_{1}} \cdots \partial x_{n}^{j_{n}}} \hat{h}_{r}^{(k)}(\boldsymbol{x})\right|_{\boldsymbol{x}=0}
$$

Thus, $\left\{\frac{h^{r}(\boldsymbol{x})}{r !}\right\}$ on a subdomain $D_{k}$ is approximated by the formal linearization function as

$$
\begin{gathered}
Y_{r}=\frac{h^{r}(\boldsymbol{x})}{r !} \approx\left[H_{r}^{(k)(10 \cdots 0)}, H_{r}^{(k)(01 \cdots 0)}, \cdots, H_{r}^{(k)\left(j_{1} \cdots j_{n}\right)},\right. \\
\left.\cdots, H_{r}^{(k)(0 \cdots N)}\right] \boldsymbol{\phi}(\boldsymbol{x})+H_{r}^{(k)(0 \cdots 0)}
\end{gathered}
$$

and a linear measurement equation with respect to $\phi$ is obtained as

$$
\begin{gathered}
\boldsymbol{Y} \approx\left[H_{r}^{(k)\left(j_{1} \cdots j_{n}\right)}\right] \boldsymbol{\phi}(\boldsymbol{x})+\left[H_{r}^{(k)(0 \cdots 0)}\right] \\
\equiv \boldsymbol{H}^{(k)} \boldsymbol{\phi}(\boldsymbol{x})+\boldsymbol{d}^{(k)}
\end{gathered}
$$

Applying Eq. (6) to Eq. (19) yields

$$
\begin{gathered}
\boldsymbol{Y} \approx \sum_{k=0}^{M}\left(\boldsymbol{H}^{(k)} \boldsymbol{\phi}(\boldsymbol{x})+\boldsymbol{d}^{(k)}\right) I_{k}(\zeta) \\
=\sum_{k=0}^{M} \boldsymbol{H}^{(k)} I_{k}(\zeta) \phi(\boldsymbol{x})+\sum_{k=0}^{M} \boldsymbol{d}^{(k)} I_{k}(\zeta)
\end{gathered}
$$

Therefore, a pseudo-formal linearization system for the measurement equation is approximately derived as

$$
\boldsymbol{Y}(t)=\tilde{H}(\zeta) \boldsymbol{z}(t)+\tilde{d}(\zeta)
$$

where

$$
\tilde{H}(\zeta)=\sum_{k=0}^{M} \boldsymbol{H}^{(k)} I_{k}(\zeta), \tilde{d}(\zeta)=\sum_{k=0}^{M} \boldsymbol{d}^{(k)} I_{k}(\zeta)
$$

To the linearized systems in Eqs. (14) and (21), the linear observer theory [10] is applied and an identity observer is obtained as

$$
\begin{gathered}
\dot{\hat{\boldsymbol{z}}}(t)=\tilde{A}(\zeta) \hat{\boldsymbol{z}}(t)+\tilde{b}(\zeta)+K(t)(\boldsymbol{Y}(t)-\tilde{H}(\zeta) \hat{\boldsymbol{z}}(t)-\tilde{d}(\zeta)) \\
=\sum_{k=0}^{M}\left\{\left(\boldsymbol{A}^{(k)} \hat{\boldsymbol{z}}(t)+\boldsymbol{b}^{(k)}\right)\right. \\
\left.+K^{(k)}(t)\left(\boldsymbol{Y}(t)-\boldsymbol{H}^{(k)} \hat{\boldsymbol{z}}(t)-\boldsymbol{d}^{(k)}\right)\right\} I_{k}(\zeta)
\end{gathered}
$$

where $\zeta=C(\hat{\boldsymbol{z}})$ and $K^{(k)}(t)$ is the observer gain on a subdomain $D_{k}$ given by

$$
K^{(k)}(t)=\frac{1}{2} P^{(k)}(t) \boldsymbol{H}^{(k)^{T}} S^{(k)}(t)
$$


$P^{(k)}(t)$ satisfies the matrix Riccati differential equation

$$
\begin{array}{r}
\dot{P}^{(k)}(t)=\boldsymbol{A}^{(k)} P^{(k)}(t)+P^{(k)}(t) \boldsymbol{A}^{(k)^{T}}+Q^{(k)}(t) \\
-P^{(k)}(t) \boldsymbol{H}^{(k)^{T}} S^{(k)}(t) \boldsymbol{H}^{(k)} P^{(k)}(t)
\end{array}
$$

where $Q^{(k)}(t), S^{(k)}(t)$ and $P^{(k)}(0)$ are arbitrary real symmetric positive definite matrices. With reference to the exponential estimator [10], the error in the estimate

$$
e=z-\hat{z}
$$

is uniformly asymptotically stable for linear systems in the sense of Lyapunov, i.e., $Q^{(k)}(t), S^{(k)}(t)$ and $P^{(k)}(0)$ are set in order that $P^{(k)}(t)$ will be real, symmetric, and positive definite for all $t \geq 0$.

From Eq. (15), the estimate $\hat{\hat{\boldsymbol{x}}}(t)$ of a nonlinear observer becomes

$$
\hat{\hat{\boldsymbol{x}}}(t)=[I, 0, \cdots, 0] \hat{\boldsymbol{z}}(t)
$$

\section{Numerical Examples}

To show the effectiveness of the approach, numerical experiments on the pseudo-formal linearization and simulations of a nonlinear observer as its application are illustrated.

\subsection{Pseudo-formal linearization}

Consider a pendulum in which the bob is connected to a rod of zero mass. Let $\theta$ denote the angle subtended by the rod and the vertical axis through the pivot point. This system is written as

$$
\frac{d^{2}}{d t^{2}} \theta(t)+a_{1} \frac{d}{d t} \theta(t)+a_{2} \sin (\theta(t))=0
$$

Setting the state variables as

$$
x_{1}=\theta, \quad x_{2}=\dot{\theta}
$$

the given system is rewritten by

$$
\dot{\boldsymbol{x}}(t)=\left[\begin{array}{c}
x_{2}(t) \\
-a_{2} \sin \left(x_{1}(t)\right)-a_{1} x_{2}(t)
\end{array}\right] \equiv \boldsymbol{f}(\boldsymbol{x}(t))
$$

In order to apply the linearization, we set $C(\boldsymbol{x})=$ $x_{1}$ because of its highest nonlinearity and consider the domain of $x_{1}$ as

$$
D=\left[-\frac{3}{4} \pi, \frac{3}{4} \pi\right]
$$

We divide this whole domain into three subdomains $(M=2)$ (see Fig. 1) as

$$
D_{0}=\left[-\frac{3}{4} \pi,-\frac{1}{4} \pi\right), D_{1}=\left[-\frac{1}{4} \pi, \frac{1}{4} \pi\right), D_{2}=\left[\frac{1}{4} \pi, \frac{3}{4} \pi\right]
$$

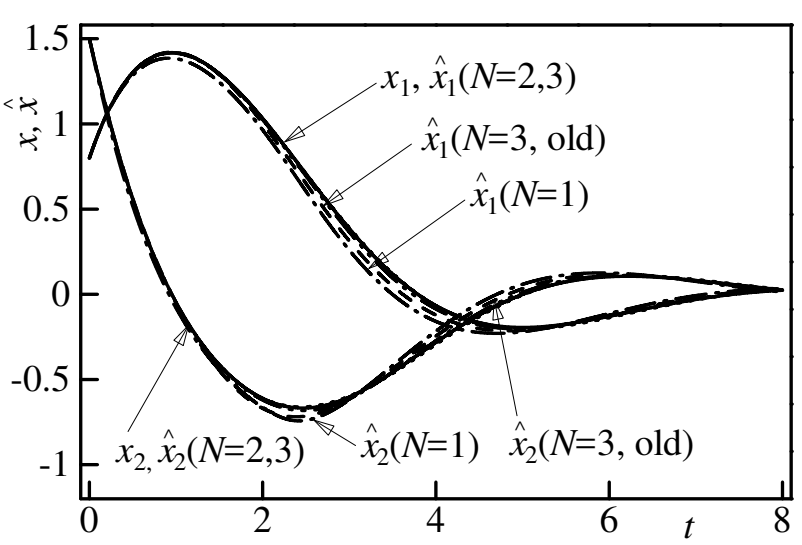

Fig.2 True value $\boldsymbol{x}$ and estimates $\hat{\boldsymbol{x}}$ with various orders

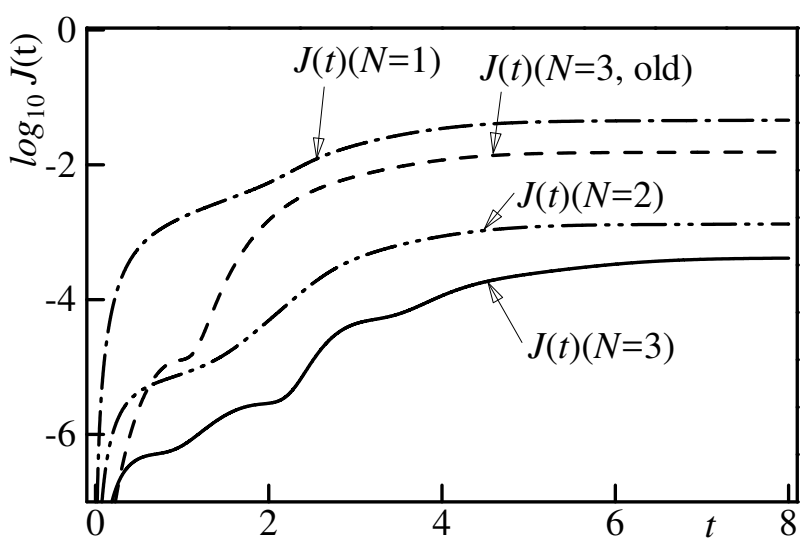

Fig.3 Integral square errors of estimation compared with previous method

The nominal operating points in Eq. (9) are set at the center point of each subdomain as

$$
\left\{\hat{x}_{10}, \hat{x}_{11}, \hat{x}_{12}\right\}=\left\{-\frac{1}{2} \pi, 0, \frac{1}{2} \pi\right\}
$$

The system parameters are set as $a_{1}=1, a_{2}=1$ and $\boldsymbol{x}(0)=[0.8,1.5]^{T}$.

Figure 2 shows the true value $\boldsymbol{x}(t)$, which is a solution of the given system (Eq. (25)), and the approximated values $\hat{\boldsymbol{x}}(t)$ given by Eq. (15) when the order of the linearization function $N$ is varied from 1 to 3 and the parameter of the automatic choosing function is set at $\mu=5$. $\hat{\boldsymbol{x}}(N=3$, old $)$ refers to a result obtained by the previous method [9] when the order of the linearization function $N$ is 3 for comparison.

To clarify the difference in the approximation errors in Fig. 2, Fig. 3 shows the integral square errors of the estimation

$$
J(t)=\int_{0}^{t}(\boldsymbol{x}(\tau)-\hat{\boldsymbol{x}}(\tau))^{T}(\boldsymbol{x}(\tau)-\hat{\boldsymbol{x}}(\tau)) d \tau
$$

for the various orders in these cases. 


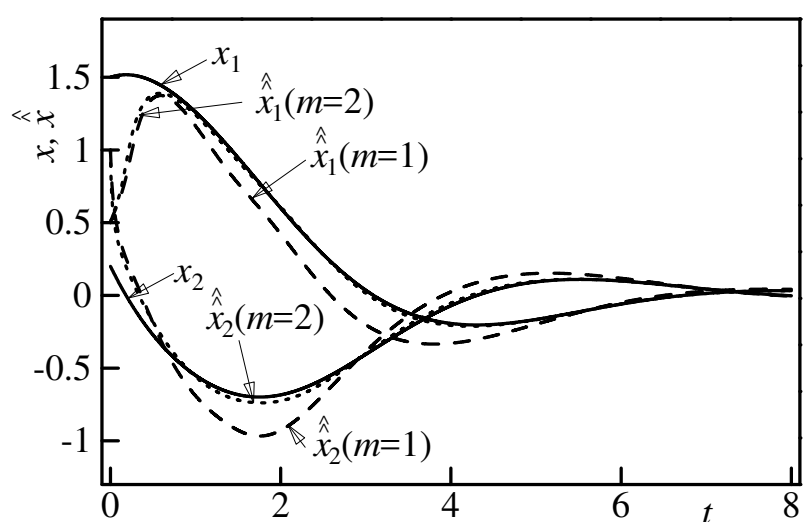

Fig.4 Nonlinear observer

\subsection{Nonlinear observer}

A nonlinear system is considered to be the same as in Eq. (26) and a measurement equation is assumed to be

$$
y=\cos \left(x_{1}(t)\right)+x_{2}(t)=h(\boldsymbol{x}(t))
$$

The subdomains, Taylor expansion points and system parameters are the same as in the previous subsection. The initial point of the system is

$$
\boldsymbol{x}(0)=[1.5,0.2]^{T}
$$

and the parameters for the nonlinear observer are set as

$$
\begin{gathered}
\hat{\hat{x}}(0)=[0.5,1]^{T}, Q^{(k)}(t)=5 I, S^{(k)}(t)=5 \\
P^{(k)}(0)=5 I(k=0,1,2), \mu=5
\end{gathered}
$$

Figure 4 shows the true value $\boldsymbol{x}(t)$ and the estimates $\hat{\hat{\boldsymbol{x}}}(t)$ in Eq. (24) obtained by the presented method when the order $N$ is 2 and $m$ is varied from 1 to 2 . Figure 5 shows the integral square errors of the estimation

$$
J(t)=\int_{0}^{t}(\boldsymbol{x}(\tau)-\hat{\hat{\boldsymbol{x}}}(\tau))^{T}(\boldsymbol{x}(\tau)-\hat{\hat{\boldsymbol{x}}}(\tau)) d \tau
$$

in this case. $N=i($ old $)(i=1,2,3)$ refers to results obtained by the previous method [9].

\section{Conclusions}

We have considered a nonlinear observer by applying a pseudo-formal linearization method of the polynomial type for both multidimensional nonlinear systems and nonlinear scalar-measurement systems. Numerical experiments show that the accuracy of this new method is improved as the order of the augmented measurement vector increases and is better than that of the previous method. It is left for future studies to extend this method to multidimensional measurement systems.

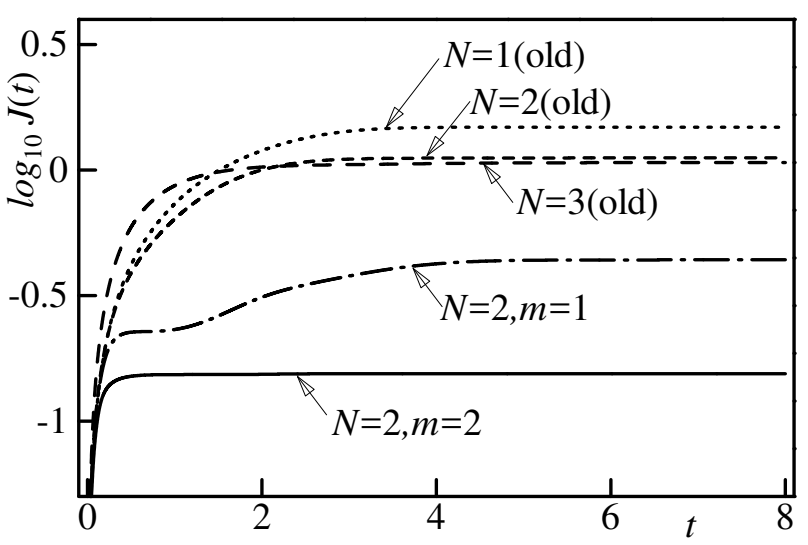

Fig.5 Integral square errors of nonlinear observer compared with previous method

\section{References}

[1] A. J. Krener: Approximate linearization by state feedback and coordinate change, Systems and Control Letters, Vol.5, pp.181-185, 1984.

[2] W. T. Baumann and W. J. Rugh: Feedback control of nonlinear systems by extended linearization, IEEE Transactions, AC-31, 1, pp.40-46, 1986.

[3] R. Marino: On the largest feedback linearizable subsystem, Systems and Control Letters, Vol.6, pp.345-351, 1986.

[4] R. R. Kadiyala: A tool box for approximate linearization on nonlinear systems, IEEE Control Systems, Vol.13, No.2, pp.47-57, 1993.

[5] A. Ishidori: Nonlinear Control Systems II, SpringerVerlag, 1999.

[6] K. Komatsu and H. Takata: Pseudo-formal linearization of polynomial type using automatic choosing function and its application to nonlinear filter, Proc. of RISP International Workshop on Nonlinear Circuits, Communications and Signal Processing, pp.121-124, 2016.

[7] H. Takata, T. Hachino, Y. Hino, K. Yunokuchi, H. Miyajima and K. Komatsu: Augmented automatic choosing control of modified filter type for nonlinear noisy measurement systems, Journal of Signal Processing, Vol.16, No.6, pp.563-569, 2012.

[8] K. Komatsu and H. Takata: Formal linearization by chebyshev interpolation for both state and measurement equations of nonlinear scalar-measurement systems and its application to nonlinear filter, Journal of Signal Processing, Vol.16, No.6, pp.263-268, 2012.

[9] K. Komatsu and H. Takata: Design of nonlinear observer by using augmented linear system based on formal linearization of polynomial type, International Journal of Computer, Electrical, Automation, Control and Information Engineering, Vol.3, No.11, pp.2523-2526, 2009.

[10] G. W. Johnson: A deterministic theory of estimation and control, IEEE Transactions on Automatic Control, Vol.14, pp.380-384, 1974. 


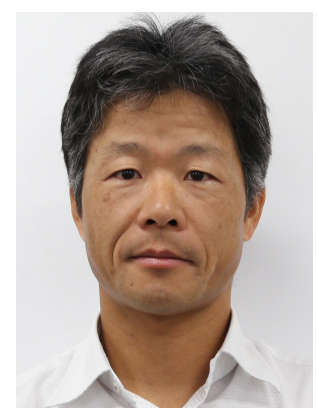

Kazuo Komatsu received his B.S. degree in computer science and Ph.D. in electrical engineering from Kyushu Institute of Technology in 1985 and 1995, respectively. He is currently a Professor at the Department of HumanOriented Information Systems Engineering in National Institute of Technology, Kumamoto College. His research interests include formal linearization for nonlinear systems and its applications. He is a member of the RISP.

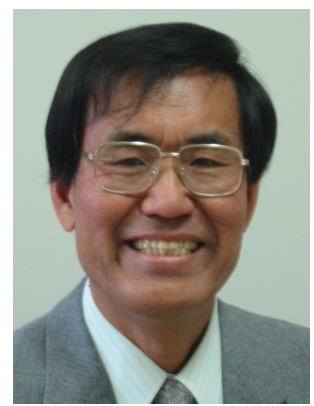

Hitoshi Takata received his B.S. degree in electrical engineering from Kyushu Institute of Technology in 1968 and his M.S. and Dr. Eng. degrees in electrical engineering from Kyushu University in 1970 and 1974, respectively. He is currently a Professor Emeritus at Kagoshima University. His research interests include the control, linearization, and identification of nonlinear systems.

(Received May 15, 2017; revised July 10, 2017) 